\title{
Caracterização da Demanda de Pacientes com Carcinoma de Colo Uterino no Hospital das Clínicas da Universidade Federal de Uberlândia, Minas Gerais, Brasil, 1984-1988
}

\author{
Characterization of Uterine Cervical Carcinoma Cases in the \\ Hospital das Clínicas, Universidade Federal de Uberlândia, \\ Minas Gerais, Brazil, from 1984 to 1988
}

\author{
Eliane Regina Pioli ${ }^{1}$ \\ Neide M. de Oliveira ${ }^{2}$ \\ Alonso G. de Rezende ${ }^{3}$
}

\begin{abstract}
PIOLI, E. R.; OLIVEIRA, N. M. \& REZENDE, A. G. Characterization of Uterine Cervical Carcinoma Cases in the Hospital das Clínicas, Universidade Federal de Uberlândia, Minas Gerais, Brazil, from 1984 to 1988. Cad. Saúde Públ., Rio de Janeiro, 9 (4): 421-427, Oct/Dec, 1993.
\end{abstract}

This is a retrospective survey of 180 cases of uterine cervical carcinoma in women admitted to the Hospital de Clinicas da Universidade Federal de Uberlândia, from 1984 to 1988, with regard to personal and temporal factors. There was a gradual increase in frequency over time and a greater frequency among women from 35 to 60 years of age. Half of the women came from other cities and states. The conditions was more frequent in multiparous women and in women who initiated sexual intercourse before the age of 20. A major proportion of women $(45.0 \%)$ had not reached menopause. Genital bleeding and abdominal pain were the most frequent clinical manifestations. Cytopathological study revealed a percentage of $60.5 \%$ of stages III and IV. There was a low frequency of in situ cancer (20.6\%), when compared to the frequency of more advanced stages (71.6 \%). The proportion of advanced stages as compared to in situ cancer increased over time, which may indicate that an optimal level of prevention was far reality in this region during the study period.

Key words: Cancer; Cervical Carcinoma; Women Health; Epidemiology

\section{INTRODUÇÃO}

O câncer cervical é uma das patologias mais comuns das vias genitais femininas, ocupando o sexto lugar como causa de morte por câncer entre as mulheres (Mackey et al., 1985). É também uma das patologias que melhor docu-

\footnotetext{
${ }^{1}$ Rua Ramiro Alves Rabelo, 68 B, Uberlândia, $M G$, 38402-069, Brasil.

${ }^{2}$ Departamento de Clínica Médica da Universidade Federal de Uberlândia. Avenida Levindo de Souza, 1.669, Uberlândia, MG, 38405-383, Brasil.

${ }^{3}$ Fundação Faculdade Regional de São José do Rio Preto. Avenida Brigadeiro Faria Lima, 5.416, São José do Rio Preto, SP, 15100-000, Brasil.
}

menta os bons resultados de um diagnóstico precoce, apresentando, com terapêutica adequada, grandes possibilidades de cura, quando detectada em seus estágios iniciais, principalmente o carcinoma in situ (Faerstein, 1987).

Atualmente, com recursos propedêuticos mais modernos, rápidos e de baixo custo, como a citopatologia e a colposcopia, a tendência é, cada vez mais, se detectar esta patologia em estágios passíveis de cura, levando à diminuição da mortalidade, como ocorre em outros países (Faerstein, 1987; Faria et al., 1983).

$\mathrm{O}$ câncer de colo uterino acomete mais freqüentemente mulheres na faixa etária de 30 a 45 anos, justamente numa idade em que as pacientes, em sua maioria, já não se preocupam 
tanto com a reprodução e não procuram regularmente o ginecologista, fator este muito importante, pois dificulta o diagnóstico precoce da patologia (Mackey et al., 1985).

O tipo histológico é, em $95 \%$ dos casos, de origem escamosa e surge na zona de transformação do colo, onde, por várias causas, poderá ocorrer a eversão do epitélio endocervical para a ectocérvice, ficando, então, o epitélio colunar simples exposto ao $\mathrm{pH}$ ácido da vagina e a outros fatores, como vírus, esmegma, espermatozóides, etc., que provavelmente estimulariam a metaplasia de tal epitélio para escamoso, no sentido de oferecer maior proteção à região. $\mathrm{E}$ aí poderá iniciar-se um processo de malignidade crescente na porção basal do epitélio, o qual pode chegar até as camadas mais superficiais, fazendo com que todo o epitélio seja tomado por células carcinomatosas (Halbe, 1987; Jones \& Jones, 1983).

Vários são os fatores que se atribuem como predisponentes para o câncer de colo, entre eles: início precoce das atividades sexuais, papilomavírus, multiparidade, higiene inadequada e infecção repetida dos órgãos genitais, além de outros mais discutidos, como o uso de anticoncepcionais na idade reprodutiva e a relação do carcinoma com o vírus herpes simples tipo 2 (Chaves et al., 1983; Halbe, 1987; Jones \& Jones, 1983).

No Brasil, principalmente no interior, a detecção do câncer de colo em estágios iniciais ainda não é muito satisfatória (Faerstein, 1987; Faleiros et al., 1987). No sentido de verificar a freqüência, os fatores de risco e o estadiamento na época do diagnóstico, realizou-se um levantamento dos casos de câncer de colo uterino diagnosticados no Hospital das Clínicas da Universidade Federal de Uberlândia (HCUFU), durante o período de 1984 a 1988.

\section{METODOLOGIA}

Realizou-se um estudo retrospectivo de 180 casos de câncer de colo uterino diagnosticados ou encaminhados para o HCUFU, no período de janeiro de 1984 a dezembro de 1988, através do estudo dos prontuários das pacientes que demandaram o serviço e que foram internadas durante este período. O HCUFU é um hospital de abrangência regional, possuindo cerca de 400 leitos. A listagem das pacientes foi obtida no Núcleo de Processamento de Dados da Universidade Federal de Uberlândia (NUPRO/ UFU). Foram excluídas do estudo as pacientes cujo diagnóstico foi feito em outro serviço, sendo encaminhadas ao Hospital das Clínicas apenas para seguimento.

Foram levantados e estudados os seguintes dados: número do prontuário, ano do diagnóstico, cor e idade da paciente, procedência, paridade, idade da menopausa, início do relacionamento sexual, sintomas apresentados na época do diagnóstico, resultado da citologia e do estadiamento clínico, baseado na classificação da Federação Internacional de Ginecologia e Obstetrícia (FIGO), constante no Quadro 1.

\section{RESULTADOS E DISCUSSÃO}

Observou-se um aumento de casos diagnosticados quanto ao tempo (Tabela 1). Tal fato pose ser atribuído a uma elevação da demanda ao serviço, devido ao conhecimento, por parte da população, da existência do serviço de ginecologia e obstetrícia e/ou pelo aumento real de diagnóstico da patologia.

TABELA 1. Casos de Carcinoma de Colo Uterino por Ano de Ocorrência no Hospital das Clínicas da Universidade Federal de Uberlândia, 1984 a 1988

\begin{tabular}{lcr}
\hline \hline Ano & Freqüência & $\%$ \\
\hline 1984 & 13 & 7,2 \\
1985 & 23 & 12,6 \\
1986 & 43 & 23,9 \\
1987 & 56 & 31,1 \\
1988 & 45 & 25,0 \\
\hline Total & 180 & 100,0 \\
\hline \hline
\end{tabular}

Na Tabela 2 observa-se uma maior freqüência entre 35 e 60 anos (média de 49,52 anos), o que está compatível com a literatura, que relata que o carcinoma de colo uterino ocorre mais em pacientes acima dos 40 anos (Bixby \& Vasquez, 1987; Tognini, 1988). Este fato se manteve no decorrer de todo o período estudado. 
QUADRO 1. Classificação do Carcinoma de Colo Uterino pela FIGO (Federação Internacional de Ginecologia e Obstetrícia)

\begin{tabular}{|c|c|}
\hline \multicolumn{2}{|r|}{ Estágios da FIGO } \\
\hline 0 & - Carcinoma in situ \\
\hline I & - Carcinoma limitado ao colo (a extensão ao corpo deve ser desprezada) \\
\hline Ia & - Carcinoma invasor pré-clínico, diagnosticado somente pela microscopia \\
\hline I a 1 & • Invasão microscópica mínima do estroma \\
\hline I a 2 & $\begin{array}{l}\text { - Tumor com componente invasor de } 5 \mathrm{~mm} \text { ou menos, com profundidade tomada } \\
\text { da base do epitélio, e } 7 \mathrm{~mm} \text { ou menos, com extensão horizontal }\end{array}$ \\
\hline $\mathrm{Ib}$ & - Tumor maior que T1a2 \\
\hline II & $\begin{array}{l}\text { - Carcinoma invadindo além do colo, mas não atingindo a parede pélvica } \\
\text { e/ou comprometendo o terço inferior da vagina }\end{array}$ \\
\hline IIa & • Sem invasão parametrial \\
\hline IIb & - Com invasão parametrial \\
\hline III & $\begin{array}{l}\text { - Carcinoma estendendo-se à parede pélvica e/ou comprometendo o terço inferior } \\
\text { da vagina e/ou causando hidronefrose ou rim não-funcionante }\end{array}$ \\
\hline IIIIa & - Tumor comprometendo o terço inferior da vagina, sem extensão à parede pélvica \\
\hline IIIb & $\begin{array}{l}\text { - Tumor estendendo-se à parede pélvica e/ou causando hidronefrose } \\
\text { ou rim não-funcionante }\end{array}$ \\
\hline IVa & $\begin{array}{l}\text { - Tumor invadindo a mucosa da bexiga ou reto e/ou estendendo-se além } \\
\text { da pélvis verdadeira } \\
\text { Nota: A presença de edema bolhoso não é evidência para se classificar o tumor } \\
\text { como T4. }\end{array}$ \\
\hline $\mathrm{IVb}$ & - Metástases à Distância \\
\hline
\end{tabular}

Fonte: UICC, 1989.

TA BELA 2. Casos de Carcinoma de Colo Uterino por Idade no Hospital das Clínicas da Universidade Federal de Uberlândia, 1984 a 1988

\begin{tabular}{lrr}
\hline \hline Faixa Etária & Freqüência & \multicolumn{2}{c}{$\%$} \\
\hline $25-29$ & 11 & 6,1 \\
$30-34$ & 8 & 4,5 \\
$35-39$ & 27 & 15,0 \\
$40-44$ & 22 & 12,2 \\
$45-49$ & 27 & 15,0 \\
$50-54$ & 20 & 11,1 \\
$55-59$ & 24 & 13,3 \\
$60-64$ & 11 & 6,1 \\
$65-69$ & 16 & 8,9 \\
$70 \mathrm{e}+$ & 14 & 7,8 \\
\hline Total & 180 & 100,0 \\
\hline \hline
\end{tabular}

Quanto à cor das pacientes, houve uma predominância da patologia em mulheres de cor parda, seguindo-se aquelas de cor branca e aquelas de cor negra. Considerando-se que, na população brasileira, a influência da raça negra é muito grande, os dados encontrados são inconclusivos quanto à raça em que a patologia predomina, podendo os mesmos estar ligados a outros fatores que incidem na população em geral, tais como condições sócio-econômicas e acesso da população aos serviços de saúde, entre outros.

Estudando-se os casos de carcinoma de colo uterino por procedência, observou-se que $48,3 \%$ das pacientes eram de Uberlândia; 39,4\% eram de outras cidades de Minas Gerais; e 10\% eram provenientes do Estado de Goiás; e 22\%, de outros Estados, evidenciando que o HCUFU é um hospital de referência regional. 
Segundo a paridade (Tabela 3), a maior freqüência ocorreu em mulheres que tiveram de cinco a nove filhos, seguidas daquelas com de dois a quatro filhos, verificando-se, portanto, uma maior predominância nas multíparas.

TA BELA 3. Distribuição das Pacientes Portadoras de Carcinoma de Colo Uterino segundo o Grau de Paridade, Hospital das Clínicas da Universidade Federal de Uberlândia, 1984 a 1988

\begin{tabular}{lrc}
\hline \hline $\mathrm{N}^{\circ}$ de Filhos & Frequiência & $\%$ \\
\hline Zero & 1 & 0,6 \\
1 & 9 & 5,0 \\
$2-4$ & 57 & 31,7 \\
$5-9$ & 72 & 40,0 \\
$10 \mathrm{e}+$ & 31 & 17,2 \\
s/informação & 10 & 5,5 \\
\hline Total & 180 & 100,0 \\
\hline \hline
\end{tabular}

Quanto ao início das relações sexuais, 58,3\% das pacientes iniciaram sua vida sexual antes dos 20 anos, enquanto somente $22,8 \%$ a iniciaram acima desta idade (Tabela 4), indicando uma maior freqüência de mulheres que iniciaram o relacionamento sexual em idade mais baixa (Chaves, 1983).

TABELA 4. Distribuição das Pacientes Portadoras de Carcinoma de Colo Uterino segundo a Idade de Início das Relações Sexuais, Hospital das Clínicas da Universidade Federal de Uberlândia, 1985 a 1988

\begin{tabular}{lrr}
\hline \hline $\begin{array}{l}\text { Idade } \\
\text { de Início }\end{array}$ & Freqüência & \multicolumn{2}{l}{$\%$} \\
\hline $12-13$ & 9 & 5,0 \\
$14-15$ & 30 & 16,6 \\
$16-17$ & 35 & 19,5 \\
$18-19$ & 31 & 17,2 \\
20 e + & 41 & 22,8 \\
s/informação & 34 & 18,9 \\
\hline Total & 180 & 100,0 \\
\hline \hline
\end{tabular}

Quanto à idade de ocorrência da menopausa (Tabela 5), verificou-se que grande percentagem das mulheres (45\%) ainda não tinha tido a menopausa.
TABELA 5. Distribuição das Pacientes Portadoras de Carcinoma de Colo Uterino segundo a Idade da Menopausa, Hospital das Clínicas da Universidade Federal de Uberlândia, 1985 a 1988

\begin{tabular}{lrc}
\hline \hline $\begin{array}{l}\text { Idade } \\
\text { da Menopausa }\end{array}$ & Freqüência & $\%$ \\
\hline $30-34$ & 3 & 1,7 \\
$35-39$ & 1 & 0,6 \\
$40-44$ & 15 & 8,3 \\
$45-49$ & 24 & 13,3 \\
$50-54$ & 16 & 8,9 \\
55 e + & 7 & 3,9 \\
n/menoupausa & 81 & 45,0 \\
s/informação & 33 & 18,3 \\
\hline Total & 180 & 100,0 \\
\hline \hline
\end{tabular}

Quanto aos sintomas apresentados à época do diagnóstico (Tabela 6), os principais foram sangramento genital, dor em baixo ventre e leucorréia, seguidos por ausência de sintomas, fraqueza e emagrecimento. $O$ fato de haver pacientes assintomáticas justifica a necessidade de exames periódicos para a detecção de carcinoma de colo de útero, como também os demais sintomas que apresentam proporções menores. Destaque deve ser dado às pacientes cujo sintoma é emagrecimento, o qual pode ser atribuído a um estágio mais avançado da doença.

Os resultados da citologia evidenciaram Papanicolaou de graus II a V em 71,1 \% dos casos (Tabela 7). Excluindo-se o grau II, que não é, isoladamente, indicativo de câncer (Clarke \& Anderson, 1979;

Spitzer et al., 1986), houve $60,5 \%$ de exames patológicos positivos. Em 28,9\% das pacientes não foram obtidas informações sobre a citologia, mostrando a deficiência na documentação dos prontuário, além do fato de muitas terem sido encaminhadas com citologia realizada em outro serviço de saúde.

Quanto ao estadiamento clínico (Tabela 8), observou-se estadiamento zero (carcinoma in situ) em 37 pacientes $(20,6 \%)$. Houve grande proporção de pacientes com estadiamento IIB $(31,6 \%)$, seguindo-se aquelas com estadiamento IB $(14,5 \%)$, os quais podem permitir, após cirurgia, a cura da doença. Porém, estes são 
TA BELA 6. Distribuição dos Sintomas das Pacientes Portadoras de Carcinoma de Colo Uterino segundo os Sintomas Apresentados à Época do Diagnóstico, Hospital das Clínicas da Universidade Federal de Uberlândia, 1984 a 1988

\begin{tabular}{lrr}
\hline \hline Quadro Clínico & Freqüência & \multicolumn{2}{c}{$\%$} \\
\hline Sangramento genital & 69 & 27,5 \\
Dor em baixo ventre & 44 & 17,5 \\
Modificação do ciclo & 30 & 11,9 \\
Leucorréia & 28 & 11,1 \\
Assintomáticas & 17 & 6,8 \\
Fraqueza e emagrecimento & 15 & 6,0 \\
Sangramento pós-coito & 14 & 5,6 \\
Dispareunia & 6 & 2,4 \\
Dor lombar & 6 & 2,4 \\
Outros & 6 & 2,4 \\
Queixas urinárias & 4 & 1,6 \\
s/Informação & 12 & 4,8 \\
\hline Total & 251 & $\mathbf{1 0 0 , 0}$ \\
\hline \hline
\end{tabular}

TABELA 7. Distribuição das Pacientes Portadoras de Carcinoma de Colo Uterino segundo o Resultado da Citopatologia, Hospital das Clínicas da Universidade Federal de Uberlândia, 1984 a 1988

\begin{tabular}{lcc}
\hline \hline Papanicolaou & Frequiência & $\%$ \\
\hline II & 19 & 10,6 \\
III & 18 & 10,0 \\
IV & 28 & 15,5 \\
V & 63 & 35,0 \\
s/informação & 52 & 28,0 \\
\hline Total & 180 & 100,0 \\
\hline \hline
\end{tabular}

estadiamentos que, havendo uma orientação e/ou uma rotina mais sistematizada de prevenção, podem ser encontrados com menor frequiência, assim como os estágios mais avançados (IIIA, IIIB, IV), que totalizaram 18,9\%.

Deve-se ressaltar, também, que 17 pacientes assintomáticas apresentaram estadiamento zero, IB ou IIB, o que justifica a necessidade de se promover a prevenção do carcinoma de colo uterino, através da consulta de rotina, em serviços que cubram a demanda da população feminina em idade fértil e após a menopausa.

TABELA 8. Distribuição das Pacientes Portadoras de Carcinoma de Colo Uterino segundo o Estadiamento Clínico por Ano do Diagnóstico, Hospital das Clínicas da Universidade Federal de Uberlândia, 1984 a 1988

\begin{tabular}{lccccccc}
\hline \hline Ano & 1984 & 1985 & 1986 & 1987 & 1988 & \multicolumn{2}{c}{ Total } \\
& & & & & & $\mathrm{N}^{\mathbf{0}}$ & $\%$ \\
\hline Zero & 7 & 6 & 8 & 8 & 8 & 37 & 20,6 \\
Ia & 1 & 1 & 0 & 2 & 2 & 6 & 3,3 \\
Ib & 1 & 3 & 8 & 9 & 5 & 26 & 14,5 \\
IIa & 2 & 1 & 2 & 0 & 1 & 6 & 3,3 \\
IIb & 1 & 8 & 12 & 16 & 20 & 57 & 31,7 \\
IIIa & 0 & 0 & 1 & 3 & 2 & 6 & 3,3 \\
IIIb & 0 & 2 & 6 & 4 & 4 & 16 & 8,9 \\
IV & 1 & 2 & 1 & 6 & 2 & 12 & 7,8 \\
s/informação & 0 & 0 & 5 & 8 & 1 & 14 & 7,8 \\
\hline Total & 13 & 23 & 43 & 56 & 45 & 180 & 100,0 \\
\hline \hline
\end{tabular}


Quanto ao grau de gravidade, na relação entre carcinoma invasor o carcinoma in situ, que pode ser um indicador do grau de prevenção da doença (Sierra \& Barrantes, 1988), encontrou-se grande proporção de carcinoma mais avançado. A relação carcinoma invasor/carcinoma in situ foi de 2,52 no período de 1984 a 1986, aumentando para 4,75 entre 1987 e 1988 . Este dado indica que a prevenção do carcinoma de colo uterino não atingiu níveis satisfatórios no período em que se realizou o estudo.

\section{CONCLUSÕES}

O carcinoma de colo uterino, no Hospital das Clínicas da Universidade Federal de Uberlândia, ocorreu mais freqüentemente nas faixas etárias de 35 a 60 anos, nas multíparas e em mulheres que iniciaram o relacionamento sexual antes dos 20 anos. Grande parte das mulheres (45\%) ainda não tinha tido a menopausa. Os sintomas mais encontrados foram sangramento genital e dor em baixo ventre. Cerca de $10 \%$ das pacientes eram assintomáticas, mas com estadiamento zero, IB ou IIB, o que justifica a necessidade de realização dos exames periodicamente.

Quanto ao estadiamento clínico da doença, encontrou-se uma pequena proporção de carcinoma in situ (20,5\%), se comparado com os estágios mais avançados da doença, que ocorrem em $71,6 \%$ dos casos. A relação carcinoma invasor/carcinoma in situ foi de 2,52 no período de 1984 a 1986, aumentando para 4,75 entre 1987 e 1988, o que indica não terem sido atingidos níveis satisfatórios de prevenção da doença na cidade e na região no período de estudo.

Considera-se importante a realização de uma avaliação dos programas e atividades de prevenção e controle do carcinoma de colo uterino em Uberlândia e na região, com o objetivo de melhor detectar a doença em estágios precoces.

\section{RESUMO}

PIOLI, E. R.; OLIVEIRA, N. M. \&

REZENDE, A. G. Caracterização da

Demanda de Pacientes com Carcinoma de

Colo Uterino no Hospital das Clínicas da

Universidade Federal de Uberlândia, Minas

Gerais, Brasil, 1984-1988. Cad. Saúde Públ., Rio de Janeiro, 9 (4): 421-427, out/dez, 1993.

Foi realizado um estudo retrospectivo de 180 casos de carcinoma de colo uterino internados no Hospital das Clínicas da Universidade Federal de Uberlândia, no período de 1984 a 1988, segundo variáveis pessoais e de tempo. Encontrou-se um aumento gradativo com o tempo e uma maior freqüência entre 35 e 60 anos de idade. Aproximadamente $51 \%$ das pacientes eram provenientes de outras cidades e estados. Verificou-se uma maior frequiência em multíparas e em mulheres que iniciaram sua vida sexual antes dos 20 anos. Grande parte das mulheres (45\%) ainda não tinha tido a menopausa. As manifestações clínicas mais frequientes foram sangramento genital irregular e dor em baixo ventre. A citopatologia evidenciou Papanicolaou de graus III em $60,5 \%$ dos casos. A proporção de carcinoma in situ $(20,6 \%)$ foi pequena em relação aos estágios mais avançados IIb (31,6\%), Ib (14,5\%), e IIIa, IIIb e IV $(18,9 \%)$, os quais, havendo uma orientação mais sistematizada de prevenção, podem ser encontrados com menor frequiência. A relação carcinoma invasor/carcinoma in situ foi de 2,52 no período de 1984 a 1986, aumentando para 4,75 entre 1987 e 1988, o que indica não terem sido atingidos níveis satisfatórios de prevenção da doença na cidade e na região no período estudado.

Palavras-Chave: Câncer; Carcinoma de Colo Uterino; Saúde da Mulher; Epidemiologia

\section{REFERÊNCIAS BIBLIOGRÁFICAS}

BIXBY, L. B. \& VASQUEZ, C. G., 1987. Epidemiología descritiva del cancer de mama y de cuello de útero in Costa Rica. Boletin de la Oficina Sanitaria Panamericana, 105: 483-493.

CHAVES, E. et. al., 1983. Epidemiologia do câncer de colo do útero. Jornal Brasileiro de Ginecologia, 93: 33-37. 
CLARKE, E. A. \& ANDERSON, T. W., 1979. Does screening by "pap" smears help prevent cervical cancer? The Lancet, 7: 1-4.

FAERSTEIN, E., 1987. População alvo e frequiência da detecção precoce de câncer de colo uterino. Cadernos do Instituto de Medicina Social, 1: 111-133.

FALEIROS, J. J.; PICCINI, R. X. \& FASSA, A. C. G., 1987. Avaliação da prevenção do carcinoma de colo de útero numa clínica de atenção primária à saúde. RBMGC, 1: 10-13.

FARIA, S. L.; CUNHA, L. S. M. \& CHIMINAZZO Jr., H., 1983. Radioterapia ou cirurgia no câncer inicial do colo do útero? FEM INA, 809-823.

HALBE, H. W., 1987. Tratado de Ginecologia. São Paulo: Roca.

JONES Jr., H. W. \& JONES, G. S., 1983. Carcinoma da cérvix. In: Tratado de Ginecologia (Novak, org.), $10^{\text {a }}$ ed., pp. 249-294, Rio de Janeiro: Guanabara Koogan.
MACKEY, E. V. et al., 1985. Carcinoma da cervix uterina. In: Tratado de Ginecologia, pp. 409436, Rio de Janeiro: Interamericana.

SIERRA, R. \& BARRANTES, R., 1988. Epidemiología del cancer de cuello uterino en Costa Rica, 1980-1983. Boletin de la Oficina Sanitaria Panamericana, 105: 345-351.

SPITZER, E. et al., 1986. Cervical cancer detected by cercicography in a patient with negative cervical citology. Obstet. Ginecol., 68: 68-71.

TOGNINI, P. H. F., 1988. Carcinoma in situ do colo uterino. Jornal Brasileiro de Ginecologia, 98: 325-328.

UICC (União Internacional Contra o Câncer), 1989. TNM - Classificação dos Tumores Malignos. $4^{\text {a }}$ ed., Brasília: Centro de Documentação do Ministério da Saúde. 